\title{
THE ROLE OF THE MONKEY FETUS IN THE EXCHANGE \\ OF THE WATER AND SODIUM OF THE AMNIOTIC FLUID *
}

\author{
By EMANUEL A. FRIEDMAN, MARY JANE GRAY, DONALD L. HUTCHINSON \\ AND ALBERT A. PLENTL \\ (From the Department of Obstetrics and Gynecology, Columbia University College of Physicians \\ and Surgeons, New York, N. Y., and the Department of Obstetrics and \\ Gynecology, University of California School of Medicine, \\ Los Angeles, Cal.)
}

(Submitted for publication October 23, 1958; accepted February 19, 1959)

Isotopic tracers allow for the expedient investigation of the exchange of elements or metabolic products between the maternal organism and conceptus. The quantity of carrier transferred per unit of time can be estimated from the behavior of tracers if the experimental data are subjected to the proper mathematical treatment. The general background and practical applications of the theory of multi-compartment systems to related biologic problems have recently been reviewed (1). Depending upon the aim of the investigation and the nature of the available experimental data, one can subdivide a given system into an arbitrary number of hypothetical compartments which exchange, or are assumed to exchange, their constituents at constant rates. If such a system is to be completely defined, time-activity curves for all compartments must be known and all must be accessible. Once the applicability of a certain kinetic model is established, the transfer rates can be calculated from a minimum of experimental data. It is the purpose of these investigations to test the applicability of a proposed kinetic model in order to provide the theoretical basis for further studies on clinical material.

It is known that the human fetus participates in the exchange of water and electrolytes between amniotic fluid and mother (2), but because of its inaccessibility a quantitative formulation of these mechanisms is not easily accomplished. A knowledge of the tracer concentration in fetal blood as a function of time is a prerequisite for the calculation of transfer rates. Such a time-activity curve for the fetal compartment can be determined directly on some experimental animals where

* This investigation was, in part, supported by a grantin-aid from the National Institutes of Health, Department of Health, Education and Welfare. samples of fetal blood can be obtained without disturbing the continuity of the system. The pregnant primate, a miniature model of the human maternal organism, should serve this purpose very well if it can be shown that the two species are comparable in the more pertinent aspects of water and electrolyte exchange.

For the study of these problems three sets of experiments were carried out. The first was intended to show that the transfer rates of water in and out of the amniotic fluid compartment of pregnant monkeys at term are identical within the limits of error of the method. The second was intended to estimate the ratio of water to sodium transfer and to compare this ratio with values previously reported for the human. Finally, attempts were made to determine time-activity curves for all three compartments simultaneously and to estimate the overall transfer rates involving the fetus in utero.

\section{METHODS}

a) Analytic procedures. The deuterium oxide in these body fluids was determined by the falling drop method (3). For the determination of tritium, the samples were combusted and the resulting water converted into hydrogen gas by the method of Graff and Rittenberg (4). A known amount of hydrogen was then transferred to Ballentine tubes and filled to atmospheric pressure with methane as quench gas (5). Disintegrations were measured in the proportional region and all results expressed uniformly as counts per minute (cpm) per $50 \mathrm{~mm}$. of mercury of hydrogen gas. The activity of samples containing sodium-22 was estimated with a well-type scintillation counter. The concentration of sodium ion was determined by the flame photometric technique.

b) Physiologic preparation. The operative procedures varied slightly according to the experiment. Pregnant rhesus or cynomolgus monkeys at term, as shown by $\mathrm{X}$-ray or known ages of gestation, were anesthetized 
with intravenous Nembutal in doses of about $30 \mathrm{mg}$. per $\mathrm{Kg}$. A small incision was made over Poupart's ligament, the femoral vein isolated, and a polyethylene catheter introduced in a distal direction. The proximal portion of the femoral vein was tied with silk. The uterus was delivered through a midline incision and, after identification of the placental margins, a purse-string suture was placed in the lower uterine segment or near the dome of the fundus. After puncture with a number 15 gauge needle through the area of myometrium surrounded by the purse-string suture, a multi-holed polyethylene catheter (Clay-Adams, size PE 90) was introduced into the amniotic cavity. The needle was withdrawn over the catheter and the purse-string suture tied.

In those experiments where fetal blood samples were desired, interplacental vessels were isolated as described by Reynolds, Paul and Huggett (6). After isolation of the vessels the distal portion leading to the secondary placenta was tied, the artery or vein punctured with a thinwalled 18 gauge needle containing a Clay-Adams PE 50 polyethylene catheter, and the catheter secured with two silk ties. The myometrium was approximated with a series of interrupted silk sutures. Intravascular clotting of fetal and maternal blood was prevented by the introduction of small amounts of heparin.

The total body water and total exchangeable sodium were either determined by routine procedures several

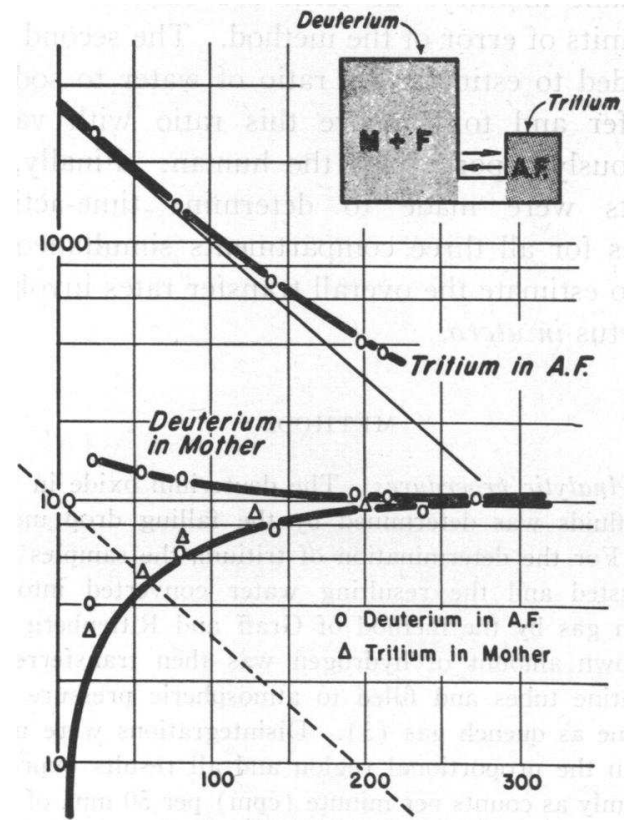

Fig. 1. Specific Activity of Tritium and Concentration of Deuterium as a Function of Time in Minutes

As shown on the kinetic model (insert) deuterium was injected into the maternal circulation and tritium introduced into the amniotic fluid. The simultaneous transfer in opposite directions can be calculated from the disappearance and appearance constants for the two tracers. days before the experiment or estimated by extrapolation of the tracer curves. The fetal body water content was estimated at 65 per cent of body weight and the volume of the amniotic fluid was determined by the Congo Red dilution method and checked by extrapolation of tracer concentration to zero time as described by Neslen, Hutchinson, Hallet and Plentl (7). Mixing of dye and tracer solutions with the amniotic fluid was accomplished mechanically by frequent withdrawal and reinjection of the fluid.

Tracers were introduced into one or more of the three compartments as described for each of the following experiments. Samples of maternal venous blood, fetal blood and amniotic fluid were obtained at intervals of five to 30 minutes over a period of three to five hours as indicated in each case. The blood samples were collected in tubes containing crystalline heparin, centrifuged and the supernatant plasma used for isotope determinations. Amniotic fluid samples were collected without special precautions and were stored in the frozen state. After a predetermined interval the experiments were terminated and the fetus delivered by cesarean section.

\section{THEORY}

Since only tracers for water and sodium were used, distribution phenomena rather than metabolic processes are considered in this study. For the mathematical treatment of the data the simplest hypothetical model is used which is not in conflict with known possibilities and representations of the system. The analysis of the data then makes it possible to calculate either the transfer rates or the size of the compartments. In the following section the theoretic background for the two- and threecompartment case is briefly discussed and a specific example is given for each.

a) The two-compartment case. First, a broad division into maternal organism and products of gestation can be made. The numerous transfers within each of them, e.g., exchanges within the fluid compartments of the maternal or fetal organism, would not appreciably influence the overall exchange mechanisms between mother, fetus and amniotic fluid provided these major exchange rates are appreciably slower. Under these circumstances the slowest, measurable rate constitutes the rate-determining step.

Wide differences in the major transfer rates may lead to further simplifications of such systems. Since there is reason to believe that the exchange rates between mother and fetus are rapid by comparison to the exchange rates between the amniotic fluid and either of the other compartments, mother and fetus may be "lumped" into one. The simplest possible system is then composed of two, rather than three compartments, exchanging their species according to the scheme:

Amniotic fluid $\rightleftarrows$ fetal + maternal systems.

There is no detectable change in the size of either compartment during the experimental period; hence, the rates in both directions must be equal. 
The calculation of the exchange rate necessitates a knowledge of : 1) the size of the combined maternal and fetal compartments, 2) the size of the amniotic fluid compartment, and 3) the disappearance constant. The experimental procedures for 1) and 2) have been described and the disappearance constant is determined graphically as follows.

After injection of the tracer into either major compartment, serial samples are taken at arbitrarily chosen intervals. The experimental points for amniotic fluid and maternal plasma which approach an equilibrium value fall on exponential curves. From a knowledge of the quantity of tracer injected, the theoretic "equilibrium" value can be calculated. Thus for the experiment illustrated graphically in Figure 1, the total body water was $3,700 \mathrm{ml}$. and the amount of deuterium oxide injected, $11.0 \mathrm{Gm}$. The theoretic equilibrium value can then be calculated as 0.297 per cent deuterium oxide by weight. Subtracting this constant from each of the experimental points gives a straight line on a semilog plot against time, the slope of which is -0.756 per hour. The volume of the amniotic fluid had been determined as $110 \mathrm{ml}$; hence the combined maternal and fetal volume is $3,590 \mathrm{ml}$. Substituting these values in Equation 1

$\rho=-\mathrm{S}_{1} \frac{\mathrm{rS}_{2}}{\mathrm{~S}_{1}+\mathrm{S}_{2}}=3,590 \frac{(0.756)(110)}{3,700}$

$=80.7 \mathrm{ml}$. per hour

a derivation of which is given in the Appendix, gives a value of $80.7 \mathrm{ml}$. or 4.5 moles per hour for the exchange rate between the two compartments.

b) The three-compartment case. The quantitative formulation of the exchanges involving the fetal system requires a more complicated theoretic system. A minimum of three compartments must be assumed, all of which could exchange with each other. Such a threecompartment system can be represented schematically.

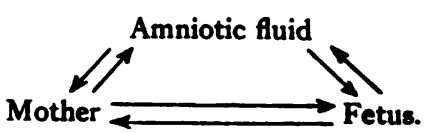

During the brief period of the experiment there is no change in size of any compartment; hence the sum of the transfer rates in and out of each compartment must be equal, i.e., the steady state conditions are met. Each of the six transfer rates may be different but the sum of these rates for each compartment must be zero. A positive value is assumed for each individual transfer rate. During the experiment no new tracer or nonisotopic material enters the system and no appreciable amount is lost, i.e., the system is "closed." Although the concentration and amount of tracer in each compartment changes with time, the total amount of tracer in all three compartments at any time is constant. At least in theory, therefore, a knowledge of two of the disappearance curves should suffice for the calculation of the third disappearance or appearance curve. The maternal compartment is more than 10 times larger than the fetal

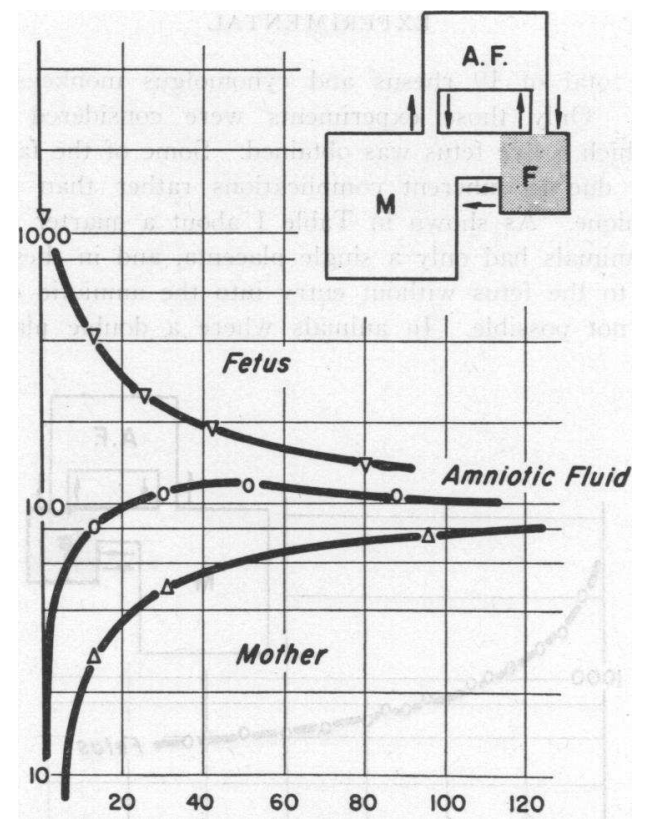

Fig. 2. Semilog Plot of the Specific Activity for Tritium in the Three Compartments as a Function of Time in Minutes

The insert shows the kinetic model, the shaded area representing the primary compartment (fetus).

or amniotic fluid compartment and this extreme difference introduces an enormous error for a time-activity curve derived in this manner. Independent measurements must, therefore, be made for each compartment.

The curves for each of the three compartments are described by the general equations:

Tracer concentration in :

$$
\begin{aligned}
\text { Amniotic fluid } & =\alpha_{1} \mathrm{e}^{r_{1} t}+\alpha_{2} \mathrm{e}^{r_{2} t}+\text { equilibrium } \\
\text { Mother } & =\beta_{1} \mathrm{e}^{r_{1} t}+\beta_{2} \mathrm{e}^{r_{2} t}+\text { equilibrium } \\
\text { Fetus } & =\gamma_{1} \mathrm{e}^{r_{1} t}+\gamma_{2} \mathrm{e}^{r_{2} t}+\text { equilibrium. }
\end{aligned}
$$

A semilog plot of the experimental data can be used to calculate the exponential constants $r_{1}$ and $r_{2}$ and the integration constants are obtained by extrapolation to zero time. As a specific illustration, the curves reproduced in Figure 2 can be expressed:

$$
\begin{aligned}
\text { Amniotic fluid } & =3740 \mathrm{e}^{-0.955 t}+75 \mathrm{e}^{-7.72 t}+100 \\
\text { Mother } & =-110 \mathrm{e}^{-0.955 t}+10 \mathrm{e}^{-7.72 t}+100 \\
\text { Fetus } & =170 \mathrm{e}^{-0.955 t}-270 \mathrm{e}^{-7.72 t}+100
\end{aligned}
$$

which defines the integration and exponential constants. The maternal body water in this particular experiment was determined as $3,470 \mathrm{ml}$., the amniotic fluid as $95 \mathrm{ml}$. and the fetal body water was, retroactively, estimated as $155 \mathrm{ml}$. By substituting these values in Equations 8, 9, 6 and 10 of the Appendix, the six individual transfer rates can be calculated. 


\section{EXPERIMENTAL}

A total of 19 rhesus and cynomolgus monkeys was used. Only those experiments were considered valid in which a live fetus was obtained. Some of the failures were due to inherent complications rather than faulty technique. As shown in Table I about a quarter of all the animals had only a single placenta, and in these access to the fetus without entry into the amniotic cavity was not possible. In animals where a double placenta

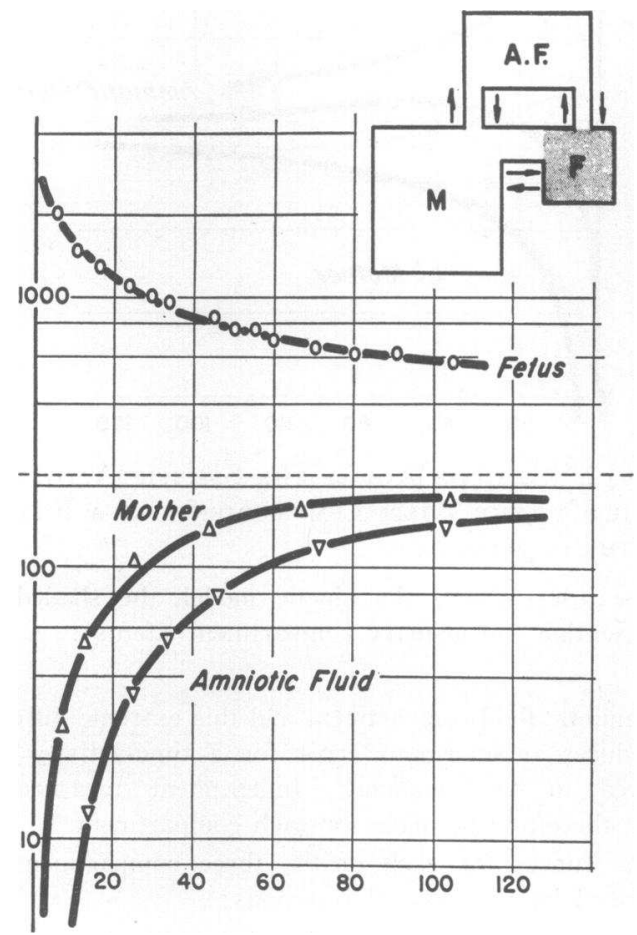

Fig. 3. Semilog Plot of Relative Radioactivity in Fetus, Mother and Amniotic Fluid as a Function of Time in Minutes Following the Injection of Sodium-22 into the Fetal Circulation

The dotted horizontal line is the calculated equilibrium value based on a knowledge of the total exchangeable sodium. The insert shows the kinetic model.
TABLE I

The incidence and location of single and double placentae in 17 pregnant rhesus and two pregnant cynomolgus monkeys

\begin{tabular}{ccc}
\hline \hline & Number & Per cent \\
\hline Single placenta & 5 & 26.3 \\
Anterior & 1 & 5.3 \\
Posterior & 4 & 21.0 \\
Double placenta & 14 & 73.7 \\
Primary anterior & 10 & 52.6 \\
Primary posterior & 4 & 21.0 \\
\hline
\end{tabular}

was encountered, the primary placenta was located on the anterior wall two-thirds of the time. Although not essential, it is nevertheless desirable to cannulate the vessels in the direction of the primary placenta since this is more likely to lead to a representative sample of fetal blood.

1) Transfers to and from the amniotic fluid. After a series of preliminary experiments to evaluate the surgical and physiological techniques, the water exchange rate of the amniotic fluid was determined using tritium and deuterium simultaneously. In the first experiment tritiated water was injected into the amniotic sac and deuterium oxide into the maternal circulation. The results of tracer analyses of amniotic fluid and maternal plasma on serial samples, both expressed on a comparable scale, are shown graphically in Figure 1 .

In five subsequent experiments sodium-22 and tritium were injected simultaneously into the amniotic sac and the transfer rates calculated in the manner described above. The significant data for each of these experiments are given in Table II.

2) Transfers involving the fetus. a) Primary compartment: Fetus. Three experiments were carried out in which the tracers were introduced into the fetal compartment by injection into the interplacental vessels. One representative experiment where tritium was employed is reproduced in Figure 2. Despite the apparently adequate number of experimental points, the error for exponential and integration constants was found to be too great for an accurate estimation of all six transfer rates. Qualitatively, the pattern was consistent in all

TABLE II

Summary of significant data on experiments designed to determine water and sodium transfer from amniotic fluid to maternal and fetal organisms

\begin{tabular}{lcccc}
\hline \hline & $\begin{array}{c}\text { Monkey } \\
\text { No.160 }\end{array}$ & $\begin{array}{c}\text { Monkey } \\
\text { No. 262 }\end{array}$ & $\begin{array}{c}\text { Monkey } \\
\text { No. 264 }\end{array}$ & $\begin{array}{c}\text { Monkey } \\
\text { M.T. }\end{array}$ \\
\hline No. 54
\end{tabular}


TABLE III

Significant data on experiments designed to determine retention curves for sodium-22 of fetal blood, defined by the expression:

$$
C_{1} e^{-r_{1} t}+C_{2} e^{-r_{2} t}+C_{3}
$$

\begin{tabular}{|c|c|c|c|}
\hline & $\begin{array}{c}\text { Monkey } \\
\text { No. 32-64 }\end{array}$ & $\begin{array}{c}\text { Monkey } \\
\text { No.32-66 }\end{array}$ & $\begin{array}{c}\text { Monkey } \\
\text { No.32-68 }\end{array}$ \\
\hline $\begin{array}{l}\text { Sodium content of fetus } \\
\quad(m E q .)\end{array}$ & 9.68 & 14.9 & 22.3 \\
\hline \multicolumn{4}{|l|}{ Retention curve for sodium: } \\
\hline $\begin{array}{l}C_{1} \\
C_{2} \\
C_{3} \\
r_{1} \text { (per hour) } \\
r_{2} \text { (per hour) }\end{array}$ & $\begin{array}{c}0.1690 \\
0.7887 \\
0.0423 \\
0.346 \\
10.39\end{array}$ & $\begin{array}{l}0.318 \\
0.629 \\
0.053 \\
0.84 \\
8.28\end{array}$ & $\begin{array}{l}0.440 \\
0.480 \\
0.080 \\
0.714 \\
6.93\end{array}$ \\
\hline $\begin{array}{l}\text { Total transfer of sodium } \\
(m E q . / h r .)\end{array}$ & 78.7 & 81.4 & 74.3 \\
\hline
\end{tabular}

three experiments. Under these conditions, the assumed closed three-compartment system leads to a time-activity curve for the amniotic fluid which passes through a maximum and eventually approaches the equilibrium value from above.

When radiosodium was injected into the fetus as the primary compartment a series of curves similar to that shown in Figure 3 was obtained. Three additional experiments were carried out in which sodium-22 and tritium were administered simultaneously into the fetal circulation and none of them yielded experimental data suitable for the calculation of all six transfer rates. Satisfactory retention curves for the fetal blood compartment were used for the calculation of combined extra- and intrafetal sodium exchange. The analysis of these retention curves is given in Table III.

b) Primary compartment: Amniotic fluid. When the amniotic fluid is chosen as the primary compartment and tritium is injected into it, the resulting disappearance curves can be used for a very accurate mathematical analysis. The experimental data on one such run are given graphically in Figure 4 and the final results on two additional experiments are presented in Table IV. The numerous operations in the graphic analysis and the calculation of each transfer rate lead to an accumulation of errors which is taken into account in the tabular presentation of the results. Although the measurements are relatively crude, the final limits of error for the majority of the transfer rates were found to be surprisingly narrow.

\section{DISCUSSION}

Based on a simplified two-compartment system an accurate estimation of the transfer rates of water and sodium from the amniotic fluid can be made. The amniotic fluid is the only compartment in this system in which "instantaneous" mixing of the tracer is possible. Except for purely mechanical complications such as an insufficient quantity

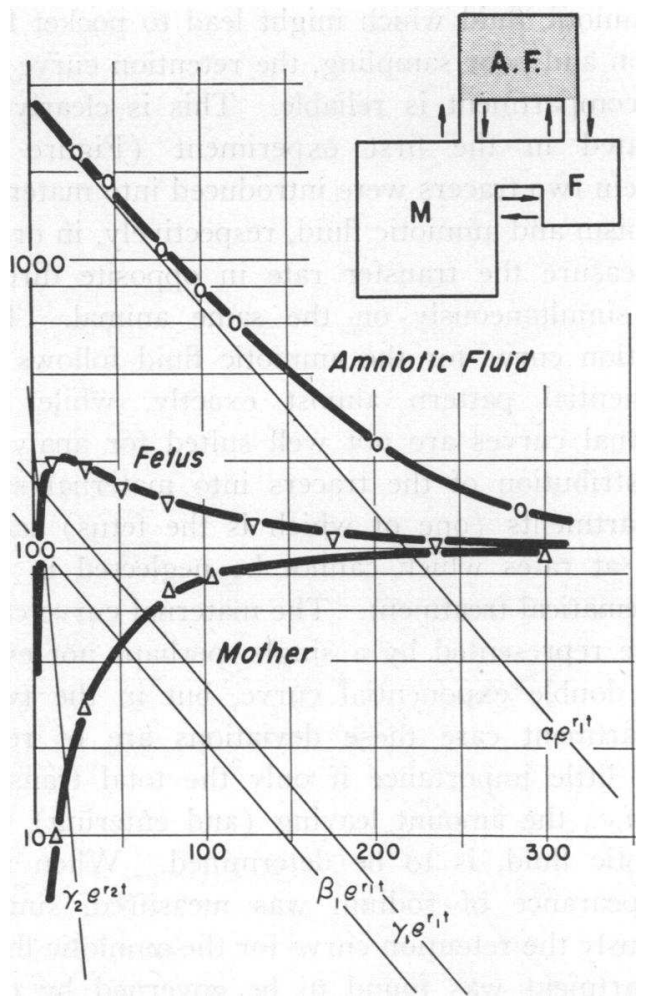

Fig. 4. Semilog Plot of Tritium Concentration Versus Elapsed Time in Minutes

The specific activity for all compartments is referred to a calculated equilibrium value arbitrarily set at 100 per cent. Insert shows the kinetic model; the shaded area the primary compartment (amniotic fluid). The straight lines represent the exponential terms from which the rate constants are calculated.

TABLE IV

Transfer rates for water as calculated from time-activity curves of maternal, fetal and amniotic fluid compartments*

\begin{tabular}{lcc}
\hline \hline & Monkey & Monkey \\
& No. 266 & 910 \\
\hline Fetus to mother & $910-930)$ & $(993-1,212)$ \\
Mother to fetus & 934 & 1,074 \\
Fetus to amniotic fluid & $(922-946)$ & $(1,018-1,130)$ \\
Amniotic fluid to fetus & 62 & 47 \\
Amniotic fluid to mother & $(43-80)$ & $(0-94)$ \\
Mother to amniotic fluid & $(51-63)$ & $(69-83)$ \\
& 11 & 12 \\
& $(0-17)$ & $(6-25)$ \\
& & $(0-87)$
\end{tabular}

* Tritiated water was injected into the amniotic fluid which served as the primary compartment. The results are tabulated in ml. per hour; the values in parentheses represent limits of error compatible with measured experimental data. 
of amniotic fluid which might lead to pocket formation and poor sampling, the retention curve for this compartment is reliable. This is clearly illustrated in the first experiment (Figure 1) wherein two tracers were introduced into maternal organism and amniotic fluid, respectively, in order to measure the transfer rate in opposite directions simultaneously on the same animal. The retention curve for the amniotic fluid follows an exponential pattern almost exactly, while the maternal curves are not well suited for analysis. A distribution of the tracers into maternal subcompartments (one of which is the fetus) takes place at rates which cannot be neglected in the mathematical treatment. The maternal curve cannot be represented by a single, perhaps not even by a double exponential curve, but in the twocompartment case these deviations are of relatively little importance if only the total transfer rate, i.e., the amount leaving (and entering) the amniotic fluid, is to be determined. When the disappearance of sodium was measured, simultaneously the retention curve for the amniotic fluid compartment was found to be governed by one exponent only. The slow distribution and equilibration of sodium in the maternal compartment makes it difficult to calculate the transfer rates in the reverse direction, i.e., from the combined maternal and fetal compartments to the amniotic fluid.

In Table $\mathrm{V}$ the mean values obtained for pregnant monkeys are compared with similar values previously reported for the human $(8,9)$. On a weight basis, the water exchange of the amniotic fluid in pregnant monkeys is about three times faster than that of humans. The exchange of sodium in the two species bears a similar relation; hence the ratio of sodium to water transfer is nearly identical. At least in this respect the two species are comparable.

There exists indirect evidence that part of the

TABLE V

Comparison of the average transfer rates for water and sodium from amniotic fluid to maternal organism in humans and monkeys at term

\begin{tabular}{lcc}
\hline \hline & Human & Monkey \\
\hline Amniotic fluid volume $(m l)$. & 785 & 107 \\
Water transfer (moles $/ \mathrm{hr})$. & 26.6 & 8.4 \\
Sodium transfer $($ moles $/ \mathrm{hr})$. & 0.0125 & 0.0047 \\
Ratio of $\mathrm{H}_{2} \mathrm{O}: \mathrm{Na}$ transfer $\times 10^{3}$ & 2.1 & 1.8 \\
& & \\
\hline
\end{tabular}

water exchange of the amniotic fluid takes place through the intermedium of the fetus. Paul, Enns, Reynolds and Chinard (10) determined the exchange of the amniotic fluid in pregnant rabbits where the fetus had been crushed in utero and compared results with those obtained in a control series. The transfer rate in the former group was about one-half that of the latter, a fact interpreted by the authors as evidence that, in the rabbit, about one-half of the transfer takes place via the fetus. The fetal membranes apparently are of less significance since tying the vitelline vessels did not affect the transfer rates. In the human, the fetus also plays an important part in this exchange (2). Within a few minutes after the injection of labeled water into the amniotic fluid of pregnant women at term, the tracer concentration in cord blood exceeds the calculated equilibrium value by a factor of two or three. If the time interval from injection of tracer to sampling of fetal blood exceeds a period of three hours, the tracer concentration in the fetus is about equal to or only slightly higher than the calculated equilibrium value. Although the exact shape of the fetal tracer concentration curve is not known, it must, at some time, pass through a maximum. It could be shown that this is only possible if more than 25 per cent of the transfer of water from amniotic fluid to mother takes place through the fetus. In the present series of experiments on monkeys the shape of the fetal curve could be determined directly and, under specific conditions, an accurate quantitative determination of the transfer rates could be made.

If it is assumed that the three compartments, amniotic fluid, mother and fetus, exchange water with each other, and if all three compartments are accessible, any one of them may serve as the primary compartment. The large size and poor initial mixing of the maternal pool make it unlikely that it would be suitable as a primary compartment if the experimental data are collected for the purpose of calculating transfer rates. On purely theoretical grounds, therefore, no attempts were made to use this approach.

Injection of tritiated water into the fetal compartment (Figure 2) gives a retention curve which falls off rapidly and soon approaches the equilibrium value. The time-activity curves for amniotic fluid and maternal compartments rise 
unequally and also approach the equilibrium value, the former from above and the latter from below. Because of the relatively large analytic error inherent in these data neither curve can be used for the accurate determination of the exponential constants. The fetal curve, on the other hand, should serve this purpose reasonably well. The initial rapid disappearance of the tracer from the fetal compartment is, to some extent, a mixing phenomenon but due mostly to the fast exchange between mother and fetus. The fetal compartment, like the maternal compartment, consists of a number of sub-compartments; these may be expected to distort the time-activity curves. Furthermore, the small difference between the experimental points and the equilibrium value near the tail end of the fetal curve renders the first exponential constant inaccurate and this, in turn, leads to a cumulative error for the second exponential constant which governs the initial sharp decline of the curve. Neither constant is reliable and the experimental data obtained by using the fetus as the primary compartment are therefore of qualitative significance only. They cannot be used for the calculation of the transfer rates within this system.

If the tracer is introduced into the amniotic fluid compartment, a mathematical analysis of the data is possible. Almost instantaneous mixing can be accomplished by physical means, virtually impossible in the two preceding cases. This results in a reliable retention curve for at least one of the three compartments; hence, the first exponential constant can be estimated with a very small margin of error. Since this constant is fixed within narrow limits, the errors for all the values derived from it are correspondingly smaller and the two integration constants for the fetal compartment can be determined with considerable accuracy. These, in turn, determine the corresponding values for the maternal compartment. An accurate knowledge of the maternal tracer concentration is, therefore, not necessary and the calculation of the transfer rates is not dependent upon such data. Moreover, the concentration of the fetal compartment throughout the most significant portion of the experiment is within the optimal range for all analytic methods. Under these conditions, the delayed mixing in the fetal compartment does not appreciably affect the values of the final transfer rates. In Table IV the six calculated transfer rates and the greatest possible errors compatible with the experimental data are given for two such experiments. The two animals were of nearly identical weight and differed only slightly in the weight of their fetuses and the volume of the amniotic fluid. The transfer rates for both animals are comparable within the experimental error of the method. Between fetus and mother there exists a molecular exchange equivalent to about $1 \mathrm{~L}$. of water per hour. On a weight basis this would correspond to about $4,200 \mathrm{ml}$. of water per hour per $\mathrm{Kg}$. of fetus, more than four times the value estimated for the human fetus at term. The total exchange of the water of the amniotic fluid, that is, the sum of its exchange with fetus and mother, corresponds to the rough estimates given above. As predicted, more than three-quarters of this exchange takes place through the fetus. Thus for monkey No. 266 the total amount of water leaving the amniotic fluid compartment could be a maximum of $68 \mathrm{ml}$. per hour and of this a minimum of 5 ( 7.3 per cent) and a maximum of $17 \mathrm{ml}$. (25 per cent). would pass directly to the mother. The limits of error for the transfer rates in the reverse direction, i.e., from fetus to amniotic fluid and mother to amniotic fluid, are somewhat broader. The direct exchange between mother and amniotic fluid, though small compared to the exchange between amniotic fluid and fetus, is nevertheless real and essential for the continuity of this system.

Numerous attempts to analyze the data of the experiments where sodium-22 was used failed to yield ranges for transfer rates suitable for interpretation. The reasons for this have been outlined above and these factors become even more significant in the mathematical treatment of the three-compartment case. The addition of several slower compartments in mother and fetus leads to a complex mathematical analysis, which is not justified on the basis of the experimental data. A direct exchange between amniotic fluid and mother is not established but probably exists. The experimental curves only indicated that there is a rapid exchange of sodium between mother and fetus and a slower one between amniotic fluid and fetus. The ratio of these exchange rates is proportional to the sodium content of the two compartments. 
If the average disappearance rate of sodium from the amniotic fluid is taken as about $4.7 \mathrm{mEq}$. per hour (Table II), the quantity exchanged between fetus and mother is probably between 60 and $70 \mathrm{mEq}$. per hour. This estimated range for extra-fetal exchange must be lower than the values calculated for the total loss of sodium from the fetal blood compartment. Injection of radiosodium into the fetal blood stream yields retention curves which can be used to calculate this loss. The retention curve is given by the expression:

$$
z=C_{1} e^{-r_{1} t}+C_{2} e^{-r_{2} t}+C_{3}
$$

where $\mathrm{z}$ is the specific activity for sodium or tritium, $\mathrm{C}_{1}, \mathrm{C}_{2}$ and $\mathrm{C}_{3}$ integration constants expressed as a fraction of one. The quantity of carrier leaving this compartment per unit of time is related to this expression by:

$$
\frac{-K}{S_{z}} z=\frac{d z}{d t}
$$

where $\mathrm{K}$ represents the sum of all transfers out, and $\mathrm{S}_{\mathbf{z}}$ the size of the compartment, i.e., its sodium or water content. Since this equation holds for all values of $z$, at $t=0, z=1$ and the total amount leaving the fetus:

$$
-\mathrm{K}=\mathrm{Sz}\left(-\mathrm{r}_{1} \mathrm{C}_{1}-\mathrm{r}_{2} \mathrm{C}_{2}\right) .
$$

\section{4}

In Table III the values obtained in this manner are compared for three animals of approximately equal weight. A total of $80 \mathrm{mEq}$. of sodium was found to leave the fetal blood stream per hour.

\section{SUMMARY}

The water and sodium exchange mechanism between mother, fetus and amniotic fluid was investigated in a series of pregnant monkeys at term. A number of likely kinetic models was assumed and their mathematical analyses reviewed. The total exchange rates for water and sodium of the amniotic fluid were determined under the assumption of closed two-compartment systems. An average of 8.4 moles of water and $4.7 \mathrm{mEq}$. of sodium were found to exchange per hour. Although the quantity exchanged is three times greater than that found for the human, the ratio of transfer rates of water and sodium is identical for the two species.
The role of the fetus was then investigated under the assumption of a closed three-compartment system, represented by amniotic fluid, fetus and mother. The data obtained in experiments where the fetus was used as the primary compartment were not sufficiently accurate for the desired calculations. Time-activity curves for the experiments where the amniotic fluid was the primary compartment were suitable for curve analysis and the six theoretic transfer rates could be calculated with a known margin of error. Between mother and fetus there exists a molecular exchange equivalent to $1,000 \mathrm{ml}$. of water per hour; between fetus and amniotic fluid about 50 ml. per hour; and the direct exchange between amniotic fluid and mother has a range of 5 to 20 $\mathrm{ml}$. per hour. The primate fetus transmits at least 75 per cent of the water leaving the amniotic fluid.

For labeled water the closed three-compartment system represents the pregnant organism reasonably well and a mathematical analysis can be applied. Tracers for sodium, on the other hand, when used in identical manner led to superficially similar conclusions but the simple closed threecompartment system does not reflect the exchange mechanisms adequately. Only a very rough estimate of the sodium exchange rates could be made. Transfer rates for sodium could not be calculated on this basis and more complex multi-compartment models will have to be worked out for this purpose.

\section{APPENDIX}

Mathematical analysis of closed steady state two- and threecompartment systems. Since access to all compartments can be assumed, the mathematical treatment of the models is relatively simple. Any combination of transfer rates for a closed three-compartment system can be expressed by the general differential equation:

$$
\begin{array}{r}
\frac{d x}{d t}+a x+b y+c z=0 \\
\frac{d y}{d t}+a_{1} x+b_{1} y+c_{1} z=0 \\
\frac{d z}{d t}+a_{2} x+b_{2} y+c_{2} z=0
\end{array}
$$

where $x, y$ and $z$ represent the specific activity or concentration of tracer in Compartments $\mathrm{X}, \mathrm{Y}$ and $\mathrm{Z}$. The coeffcients $a, b, c$, and so forth are related to the transfer rates and their definition depends upon the model under consideration.

For the three-compartment system where $\mathrm{X}, \mathrm{Y}$ and $\mathrm{Z}$ 
all exchange :

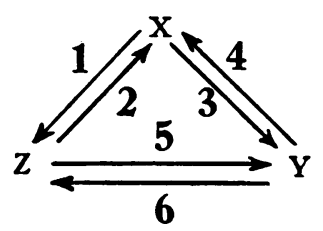

the nine coefficients are:

$$
\begin{aligned}
& \text { (1) } \quad \mathrm{a}=\frac{\rho_{1}+\rho_{3}}{\mathrm{~S}_{\mathrm{x}}} \\
& \text { (2) } \mathrm{b}=\frac{-\rho_{4}}{\mathrm{~S}_{\mathrm{x}}} \\
& \text { (3) } \mathrm{c}=\frac{-\rho_{2}}{\mathrm{~S}_{\mathrm{x}}} \\
& \text { (4) } \mathrm{a}_{1}=\frac{\rho_{3}}{\mathrm{~S}_{\mathrm{x}}} \\
& \text { (5) } \mathrm{b}_{1}=\frac{\rho_{4}+\rho_{6}}{\mathrm{~S}_{\mathrm{y}}} \\
& \text { (6) } \quad c_{1}=\frac{-\rho_{5}}{\mathrm{~S}_{\mathrm{y}}} \\
& \text { (7) } \mathrm{a}_{2}=\frac{-\rho_{1}}{\mathrm{~S}_{\mathrm{z}}} \\
& \text { (8) } \quad \mathrm{b}_{2}=\frac{-\rho_{6}}{\mathrm{~S}_{\mathrm{z}}} \\
& \text { (9) } \quad \mathrm{c}_{2}=\frac{\rho_{2}+\rho_{5}}{\mathrm{~S}_{\mathrm{z}}}
\end{aligned}
$$

where the $\rho$ 's are the transfer rates in the specific directions defined above and $S_{x}, S_{y}$ and $S_{z}$ the size of Compartments $\mathrm{X}, \mathrm{Y}$ and $\mathrm{Z}$. The experimental data are given in terms of curves showing the change of $x, y$ and $z$ as a function of time. These curves are the solution of the general differential equation and the treatment of data is concerned with the derivation of coefficients (1) to (9). The measurable quantities are double exponential equations representing the change of $\mathrm{x}, \mathrm{y}$ and $\mathrm{z}$ with respect to time:

$$
\begin{aligned}
& \mathrm{x}=\alpha_{1} \mathrm{e}^{\mathrm{r}_{1} \mathrm{t}}+\alpha_{2} \mathrm{e}^{\mathrm{r}_{2} \mathrm{t}}+\alpha_{3} \\
& \mathrm{y}=\beta_{1} \mathrm{e}^{\mathrm{r}_{1} \mathrm{t}}+\beta_{2} \mathrm{e}^{\mathrm{r}_{2} \mathrm{t}}+\beta_{3} \\
& \mathrm{z}=\gamma_{1} \mathrm{e}^{\mathrm{r}_{1} \mathrm{t}}+\gamma_{2} \mathrm{e}^{\mathrm{r}_{2} \mathrm{t}}+\gamma_{3} .
\end{aligned}
$$

Graphic analysis of these curves gives the values for the exponential constants $r_{1}$ and $r_{2}$ and the integration constants $\alpha_{1}, \alpha_{2}, \beta_{1}, \beta_{2}, \gamma_{1}$ and $\gamma_{2}$. The three constants $\alpha_{3}$, $\beta_{3}$ and $\gamma_{3}$ are identical and equal to the equilibrium value in whatever units $x, y$ and $z$ are expressed. The relation of the coefficients and the measurable constants is found by solving the original differential equation. For this solution it is assumed that each variable can be expressed as the sum of one or more exponential terms:

$$
\mathrm{x}=\mathrm{e}^{\mathrm{rt}} ; \mathrm{y}=\mathrm{e}^{\mathrm{rt}} ; \quad \mathrm{z}=\mathrm{e}^{\mathrm{rt}} \text {. }
$$

Differentiating each of these terms and substituting in 5 gives:

$$
\begin{array}{r}
(a+r) \alpha+b \beta+c \gamma=0 \\
a_{1} \alpha+(b+r) \beta+c \gamma=0 \\
a_{2} \alpha+b_{2} \beta+\left(c_{2}+r\right)=0 .
\end{array}
$$

There are three integration and three exponential constants, hence nine equations or three sets of the form 8 . From these nine equations the nine coefficients can be calculated or expressed in terms of the measured constants; the following relations are useful for the problem under consideration:

$$
\begin{aligned}
& \mathrm{a}_{1}=\frac{\frac{-\mathrm{r}_{1} \beta_{1}}{\beta_{1}-\gamma_{1}}+\frac{\mathrm{r}_{2} \beta_{2}}{\beta_{2}-\gamma_{2}}}{\frac{\alpha_{1}-\gamma_{1}}{\beta_{1}-\gamma_{1}}-\frac{\alpha_{2}-\gamma_{2}}{\beta_{2}-\gamma_{2}}} \\
& \mathrm{c}_{1}=-\frac{\mathrm{r}_{1} \beta_{1}}{\gamma_{1}-\beta_{1}}-\mathrm{a} \frac{\alpha_{1}-\beta_{1}}{\gamma_{1}-\beta_{1}} \\
& \mathrm{a}_{2}=\frac{\frac{-\mathrm{r}_{2} \gamma_{2}}{\gamma_{2}-\beta_{2}}+\frac{\mathrm{r}_{1} \gamma_{1}}{\gamma_{1}-\beta_{1}}}{\frac{\alpha_{1}-\beta_{2}}{\gamma_{2}-\beta_{2}}-\frac{\alpha_{1}-\beta_{1}}{\gamma_{1}-\beta_{1}}} \\
& \mathrm{c}_{2}=\frac{-\mathrm{r}_{1} \gamma_{1}}{\gamma_{1}-\beta_{1}}+\mathrm{a}_{2} \frac{\beta_{1}-\alpha_{1}}{\gamma_{1}-\beta_{1}} \\
& \mathrm{c}=\frac{\frac{\mathrm{r}_{1} \alpha_{1}}{\beta_{1}-\alpha_{1}}-\frac{\mathrm{r}_{2} \alpha_{2}}{\beta_{2}-\alpha_{2}}}{\frac{\gamma_{2}-\alpha_{2}}{\beta_{2}-\alpha_{2}}-\frac{\gamma_{1}-\alpha_{1}}{\beta_{1}-\alpha_{1}}}
\end{aligned}
$$

Because of the steady state conditions (the sum of all transfer rates in and out of each compartment is equal to zero) only four of the six coefficients need be calculated directly from the constants, the remaining transfer rates being defined:

$$
\begin{aligned}
& \rho_{1}+\rho_{6}=\rho_{2}+\rho_{5} \\
& \rho_{2}+\rho_{4}=\rho_{1}+\rho_{3} \\
& \rho_{3}+\rho_{5}=\rho_{4}+\rho_{6} .
\end{aligned}
$$

The analysis of a simplified system can be accomplished in essentially the same manner. If, for example, there is no pathway from mother to amniotic fluid, a catenary system of the type:

$$
\mathrm{X} \stackrel{\rho_{1}}{\rightleftarrows} \mathrm{\rho _{2 }} \underset{\mathrm{Z}}{\rightleftarrows} \mathrm{Y}
$$

represents the kinetic model. With reference to the general differential Equation 5 the coefficients $b$ and $a_{1}$ are equal to zero, i.e., the transfer rates $\rho_{3}$ and $\rho_{4}$ do not exist. Since the system is in a steady state, $a=-c$. Substituting in Equation 5 gives:

$$
\mathrm{a} \alpha_{1}+\mathrm{r} \alpha_{1}+\mathrm{c} \gamma_{1}=0
$$

and

$$
c=\frac{r_{1} \alpha_{1}}{\gamma_{1}-\alpha_{1}} \text { and } \rho_{1}=\frac{-r_{1} \alpha_{1} S_{x}}{\gamma_{1}-\alpha_{1}} .
$$

For the two compartment case, where mother and fetus are "lumped"

$$
\mathrm{X} \rightleftarrows(\mathrm{Y}+\mathrm{Z})
$$

the general equation takes the form:

$$
\begin{gathered}
\frac{d x}{d t}+a x+b y=0 \\
\frac{d y}{d t}+a_{1} x+b_{1} y=0 .
\end{gathered}
$$


Differentiating the assumed solutions:

$$
\mathrm{x}^{\circ}=\mathrm{e}^{\mathrm{rt}} \text { and } \mathrm{y}=\mathrm{e}^{\mathrm{rt}}
$$

and substituting in Equation 13 one obtains:

$$
\begin{array}{r}
(a+r) \alpha_{1}+b \beta_{1}=0 \\
a_{1} \alpha_{1}+\left(b_{1}+r\right) \beta_{1}=0
\end{array}
$$

where the coefficients $a, b, a_{1}$ and $b_{1}$ are related to the transfer rate

and

$$
\begin{gathered}
a=-b=\frac{\rho}{S_{x}} \\
a_{1}=-b_{1}=-\frac{\rho}{S_{y}}
\end{gathered}
$$$$
\mathrm{a} \alpha_{1}+\mathrm{r} \alpha_{1}-\mathrm{a} \beta=\mathrm{a}_{1} \alpha_{1}-\mathrm{a}_{1} \beta_{1}+\mathrm{r} \beta
$$

$$
\rho=-S_{x} \frac{r \alpha}{\alpha-\beta}=+S_{y} \frac{r \beta}{\alpha-\beta}
$$

which is identical with the Sheppard and Householder (11) equation since $\alpha$ and $\beta$ have opposite signs and are proportional to the size of the two compartments.

\section{REFERENCES}

1. Robertson, J. S. Theory and use of tracers in determining transfer rates in biological systems. Physiol. Rev. 1957, 37, 133.

2. Gray, M. J., Neslen, E. D., and Plentl, A. A. Estimation of water transfer from amniotic fluid to fetus. Proc. Soc. exp. Biol. (N. Y.) 1956, 92, 463.

3. Fetcher, E. S., Jr. Modifications of apparatus for deuterium oxide determination by the falling drop. Industr. engin. Chem. (Analyt. Ed.) 1944, 16, 412.
4. Graff, J., and Rittenberg, D. Microdetermination of deuterium in organic compounds. Analyt. Chem. 1952, 24, 878.

5. Bernstein, W., and Ballentine, R. Gas phase counting of low energy beta-emitters. Rev. Sci. Instr. 1950, 21, 158.

6. Reynolds, S. R. M., Paul, W. M., and Huggett, A. St. G. Physiological study of the monkey fetus in utero: A procedure for blood pressure recording. blood sampling, and injection of the fetus under normal conditions. Bull. Johns Hopk. Hosp. 1954, 95, 256.

7. Neslen, E. D., Hutchinson, D. L., Hallet, R. L., and Plentl, A. A. Dilution methods for determination of amniotic fluid volume. Obstet. and Gynec. 1954, 3, 598.

8. Hutchinson, D. L., Hunter, C. B., Neslen, E. D., and Plentl, A. A. The exchange of water and electrolytes in the mechanism of amniotic fluid formation and the relationship to hydramnios. Surg. Gynec. Obstet. 1955, 100, 391.

9. Neslen, E. D., Hunter, C. B., and Plentl, A. A. Rate of exchange of sodium and potassium between amniotic fluid and maternal system. Proc. Soc. exp. Biol. (N. Y.) 1954, 86, 432.

10. Paul, W. M., Enns, T., Reynolds, S. R. M., and Chinard, F. P. Sites of water exchange between the maternal system and the amniotic fluid of rabbits. J. clin. Invest. 1956, 35, 634.

11. Sheppard, C. W., and Householder, A. S. The mathematical basis of interpretation of tracer experiments in closed steady-state systems. J. appl. Physics 1951, 22, 510. 